\title{
ESTIMATION OF PARAMETERS IN A PLANAR SEGMENT PROCESS WITH A BIOLOGICAL APPLICATION
}

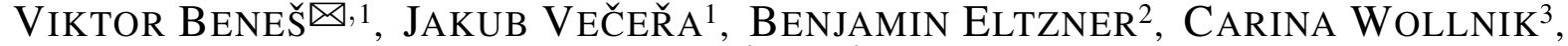 \\ FLORIAN REHFELDT ${ }^{3}$, VERONIKA KRÁlOVÁ ${ }^{1}$ AND STEPHAN HUCKEMANN ${ }^{2}$ \\ ${ }^{1}$ Charles University, Faculty of Mathematics and Physics, Department of Probability and Mathematical \\ Statistics, Sokolovská 83, 18675 Praha 8, Czech Republic ; ${ }^{2}$ Georg-August-University of Göttingen, Institute of \\ Mathematical Stochastics, Goldschmidtstrasse 7, 37077 Göttingen, Germany; ${ }^{3}$ Georg-August-University of \\ Göttingen, Third Institute of Physics - Biophysics, Friedrich-Hund-Platz 1, 37077 Göttingen, Germany \\ e-mail: benesv@karlin.mff.cuni.cz, vecera@karlin.mff.cuni.cz, beltzne@uni-goettingen.de, \\ carina.wollnik@phys.uni-goettingen.de, florian.rehfeldt@phys.uni-goettingen.de,veronika.kralo@gmail.com, \\ huckeman@mathematik.uni-goettingen.de
}

(Received September 18, 2016; revised November 17, 2016; accepted November 30, 2016)

\begin{abstract}
The paper deals with modeling of segment systems in a bounded planar set (a cell) by means of random segment processes. Two models with a density with respect to the Poisson process are presented. In model I interactions are given by the number of intersections, model II includes the length distribution and takes into account distances from the centre of the cell. The estimation of parameters of the models is suggested based on Takacz-Fiksel method. The method is tested first using simulated data. Further the real data from fluorescence imaging of stress fibres in mesenchymal human stem cells are evaluated. We apply model II which is inhomogeneous. The degree-of-fit testing of the model using various characteristics yields quite satisfactory results.
\end{abstract}

Keywords: parameter estimation, random segment process, stem cell, stress fibre.

\section{INTRODUCTION}

In stochastic geometry (Chiu et al., 2013) random geometrical objects and their systems are studied. The basic model is a stationary spatial point process on the whole Euclidean space. Sometimes, however, a stochastic model in a bounded set is needed, e.g. in the biological example investigated in this paper. We will study the distribution of actin stress fibres in individual stem cells. The goal is to model this system for each cell by means of a finite point process (Møller and Waagepetersen, 2007; Baddeley, 2007) including marks which leads to a segment process (Chiu et al., 2013; Pawlas, 2014).

The goal of our present paper splits into two aims. First we develop background theory of segment processes. Particularly we are interested in parameter estimation in models given by a density with respect to the Poisson process (Møller and Waagepetersen, 2004). For systems of objects the disc process case was developed in Møller and Helisova $(2008 ; 2010)$, the facet process in Večeřa and Beneš (2016); Večeřa (2016), where facets are compact subsets of hyperplanes in $\mathbb{R}^{d}$. Because of problems with normalizing constant the Takacz-Fiksel method or maximum pseudolikelihood method are suitable choices, cf. Coeurjolly et al. (2012) for spatial point processes and Dereudre et al. (2014) for disc processes. The applicability of the TakaczFiksel method is first successfully tested on simulated data. The simulation of a realization of a finite segment process is obtained by means of the birthdeath Metropolis-Hastings algorithm of Markov chain Monte Carlo (Geyer and Møller, 1994).

The second aim of the paper consists in an application of the suggested methods to the real data from fluorescence imaging of stress fibres in adult human mesenchymal stem cells. The stem cells have been cultured on gels for a time span of 24 hours. We evaluate especially the cells on a low stiffness gel since they present more randomness and less inhomogeneity than the others. Using the Filament Sensor (Eltzner et al., 2016) algorithm it is possible to transform the raw data onto a system of segments. Here, the true window corresponds to a single cell which is roughly approximated by an ellipse. The parameters of a suitable model with density with respect to a Poisson segment process are estimated. The degree of fit of real data with the model is then tested using various statistics by means of Monte-Carlo testing. 


\section{MATERIALS AND METHODS}

In many applications, systems of randomly dispersed segments in the plane or space are investigated. In biology, such systems occur e. g. when using fluorescence imaging to observe stress fibres in stem cells. Real data from an ongoing research consists of actin stress fibres in human mesenchymal stem cells (hMSCs) taken from the bone marrow. In the experiment, stem cells have been cultured on gels of different stiffness for 24 hours. This stiffness is given in terms of the Young's modulus, the ratio of stress by strain, i.e. the force per area needed to deform the material.

Earlier experiments have found that hMSCs can be mechanically guided to differentiate towards various cell types depending on the substrate elasticity they are grown on, namely neuron precursor cells for $1 \mathrm{kPa}$, muscle precursor cells for $10 \mathrm{kPa}$ and bone precursor cells for $30 \mathrm{kPa}$ (Engler et al., 2006). Especially the differentiation into neuron precursor cells is remarkable, since hMSC stem from the mesodermal tissue layer, while neurons are ectodermal cells. It has also been found that these three populations of cells on different gels express significantly disparate fibre patterns after 24 hours on the gel, (Zemel et al., 2010). It is therefore interesting to closely examine the stress fibre patterns especially for cells on a gel with $1 \mathrm{kPa}$ stiffness. In the present paper we investigate group $G 1$ of $n_{1}=138$ cells which corresponds to a Young's modulus of $1 \mathrm{kPa}$ and it is mostly suitable for a simple stochastic modeling.

Using the Filament Sensor algorithm (Eltzner et al., 2016) it is possible to transform the raw data into a system of segments. Fig. 1 shows an example cell and the automatic line detection result. The corresponding segment systems of each cell are characterized by the following geometrical parameters:

- cell shape,

- spatial distribution of segments,

- length distribution,

- directional distribution.

In the following we suggest a methodology of quantitative description of these attributes.
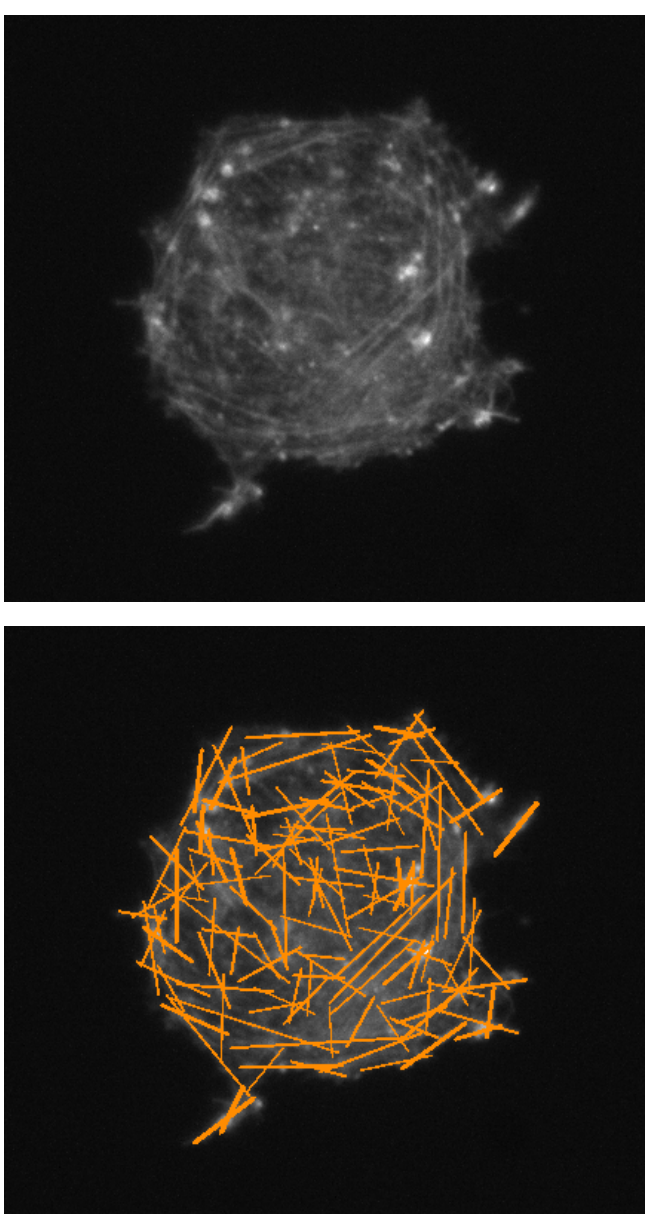

Fig. 1. Cell 12 on $1 \mathrm{kPa}$ gel, original microscopy image (upper image) and microscopy image overlayed with fibers (lower image) detected by the Filament Sensor (Eltzner et al., 2016).

\section{DESCRIPTION OF THE CELL SHAPE}

The cell shape is determined automatically by the Filament Sensor. From the raw shape, the program derives the center point, the area and the aspect ratio of the cell. This yields a simple approximation of the shape by an ellipse. An elliptical window with axes $e_{1} \geq e_{2}$ can be realized in our simulations, however this suggests restricting attention to cells whose real shape closely fits the elliptical approximation. For the example cell from Fig. 1 we illustrate the corresponding elliptical approximation in Fig. 2. If $A$ is the cell and $B$ is the ellipse, the number of pixels $\operatorname{card}(B \backslash A)=\operatorname{card}(A \backslash B)$ and this number is minimized. For the cells considered in our study, less than $10 \%$ of the cells' pixels lie outside the ellipse. Of the segment pixels even less than 3\% lie outside the ellipse for every image. For these images we can therefore consider the elliptical approximation of the cell shape as sufficient and to accept an assumption that the segments are completely included in the ellipse. 


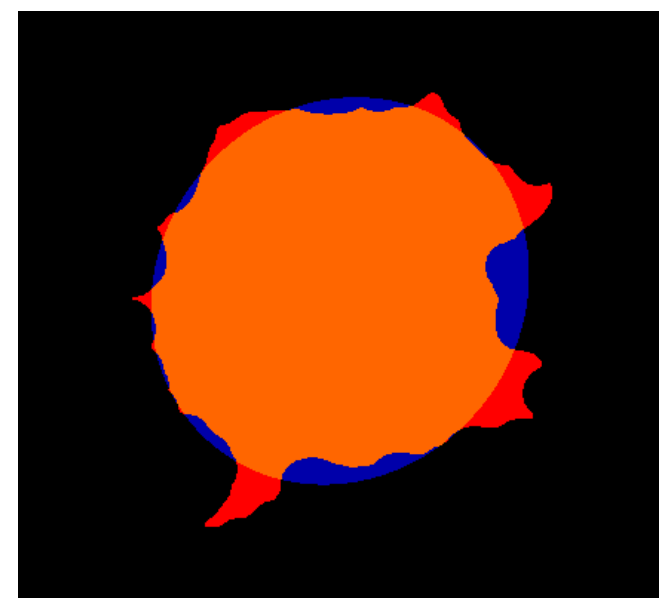

Fig. 2. The shape of cell 12 on $1 \mathrm{kPa}$ gel as detected by the Filament Sensor (Eltzner et al., 2016) compared to the elliptical approximation.

\section{SPATIAL POINT PROCESS GIVEN BY A DENSITY}

Consider a bounded measurable planar set $B \subset \mathbb{R}^{2}$ of area $|B|>0$ and a measurable space $(\mathbf{N}, \mathscr{N})$ of finite point subsets of $B$. Here $\mathbf{N}$ is the space of outcomes and $\mathscr{N}$ a system of measurable subsets of $\mathbf{N}$, see Baddeley (2007) for more details. A random element having values in $(\mathbf{N}, \mathscr{N})$ is called a finite point process. Let a Poisson point process $\eta$ on $B$ have finite intensity measure $\lambda$ (mean number of points in a given subset of $B$ ) and probability distribution $P_{\eta}$ on $\mathscr{N}$. We consider a finite point process $\mu$ on $B$ given by a density $p$ with respect to $P_{\eta}$, i. e. with distribution $P_{\mu}$

$$
\mathrm{d} P_{\mu}(\mathbf{x})=p(\mathbf{x}) \mathrm{d} P_{\eta}(\mathbf{x}), \mathbf{x} \in \mathbf{N},
$$

where $p: \mathbf{N} \rightarrow \mathbb{R}_{+}$is measurable satisfying

$$
\int_{\mathbf{N}} p(\mathbf{x}) \mathrm{d} P_{\eta}(\mathbf{x})=1 \text {. }
$$

For a measurable map $F: \mathbf{N} \rightarrow \mathbb{R}, F(\mu)$ is a random variable. The distribution of the process is alternatively determined by the conditional intensity,

$$
\lambda^{*}(\mathbf{x}, u)=\frac{p(\mathbf{x} \cup u)}{p(\mathbf{x})},
$$

where $\lambda^{*}(\mathbf{x}, u)|\mathrm{d} u|$ is interpreted as the probability that there is a point of the process in a small neighbourhood $\mathrm{d} u$ of $u$ given that the process is equal to $\mathbf{x}$ outside $\mathrm{d} u$. An important tool is the Georgii-Nguyen-Zessin formula

$$
\mathbb{E}\left[\sum_{u \in \mu} q(u, \mu \backslash\{u\})\right]=\int_{B} \mathbb{E}\left[\lambda^{*}(\mu, u) q(u, \mu)\right] \mathrm{d} u,
$$

valid for any measurable test function $q$ on $B \times \mathbf{N}$.

\section{THE SEGMENT PROCESS WITH REFERENCE DIRECTIONAL DISTRIBUTION}

Let $B \subset \mathbb{R}^{2}$ be as above, let $[0, \pi)$ be the semicircle of axial directions, and put

$$
Y=B \times[0, \pi) .
$$

A segment $x=(s, \phi) \in Y$ has centre $s$ and direction $\phi$ (we will assume for simplicity a fixed segment length $r>0$ here).

Consider a measurable space $(\mathbf{M}, \mathscr{M})$ of finite point sets in $Y$. A random element having values in $(\mathbf{M}, \mathscr{M})$ is called a finite segment process.

We deal with the Poisson segment process $\eta(s)$ with the intensity measure $\lambda$ on $Y$, where

$$
\lambda(\mathrm{d}(y, \phi))=\frac{s}{\pi} \mathrm{d} y \mathrm{~d} \phi
$$

for a constant $s>0$. Let the segment process $X$ have a density $p$ w.r.t. $\eta(1)$, we consider model I:

$$
p(\mathbf{x})=c \exp (a N(\mathbf{x})) z^{n(\mathbf{x})} \prod_{x_{i} \in \mathbf{x}} g\left(\phi_{i}\right),
$$

with parameters $a \leq 0, z>0$, the normalizing constant $c$, the statistics $n(\mathbf{x})$ (the total number of segments in $\mathbf{x}), N(\mathbf{x})$ (the total number of intersections between segments in $\mathbf{x}$ ). Finally $\phi_{i}$ is the direction of $i$-th segment $x_{i}$ and $g$ is a reference probability density on $[0, \pi)$. The conditional intensity corresponding to the density $p$ in (4) is for $u=(y, \phi), u \notin \mathbf{x}$,

$$
\lambda^{*}(\mathbf{x}, u)=z g(\phi) \exp \left(a N_{\mathbf{x}}(u)\right),
$$

where $N_{\mathbf{x}}(u)$ is the number of segments from $\mathbf{x}$ which hit $u$. The Metropolis-Hastings birth-death algorithm for simulating a realization of the segment process $X$ is that of Geyer and Møller (1994) where the Hastings ratio for birth is

$$
H(\mathbf{x}, u)=\exp \left(a N_{\mathbf{x}}(u)\right) \frac{z g(\phi)|B|}{(n(\mathbf{x})+1)}
$$

and the proposal density is uniform on $Y$.

Example: Let the directional density $g$ be that of von Mises distribution on $[0, \pi)$ with parameters $\kappa \geq$ $0, v \in \mathbb{R}$, which is suitable for unimodal distribution ( $v$ is the mode and $\kappa$ reflects the concentration around $v)$ :

$$
g(\phi)=d(\kappa) \exp (\kappa \cos (2(\phi-v))), \quad \phi \in[0, \pi],
$$


$d(\kappa)=\frac{1}{\pi I_{0}(\kappa)}, I_{0}(\kappa)$ is the modified Bessel function of the first class and order 0 . Then

$$
p(\mathbf{x})=c(\boldsymbol{\theta}) \exp (\langle\boldsymbol{\theta}, G(\mathbf{x})\rangle), \quad \mathbf{x} \in \mathbf{M},
$$

where $\langle.,$.$\rangle is the scalar product,$

$$
\begin{aligned}
\boldsymbol{\theta} & =(a, \log (z d(\kappa)), \kappa), \\
G(\mathbf{x}) & =\left(N(\mathbf{x}), n(\mathbf{x}), \sum_{x_{i} \in \mathbf{x}} \cos \left(2\left(\phi_{i}-v\right)\right)\right),
\end{aligned}
$$

segments $x_{i}=\left(s_{i}, \phi_{i}\right)$ have centres $s_{i} \in B$ and directions $\phi_{i} \in[0, \pi)$. The normalizing constant $c(\theta)$ is defined as

$$
c(\boldsymbol{\theta})=\frac{1}{\int \exp \{\langle\boldsymbol{\theta}, G(\mathbf{x})\rangle\} \mathrm{d} P_{\eta(1)}(\mathbf{x})} .
$$

Also

$$
\left\{\theta \in \mathbb{R}^{3}: \int \exp \{\langle\theta, G(\mathbf{x})\rangle\} \mathrm{d} P_{\eta(1)}(\mathbf{x})<\infty\right\}
$$

is the largest set of $\theta$ such that the density (Eq. 5) is well defined. It is the exponential class density (Møller and Waagepetersen, 2004) where classical maximum likelihood estimation (MLE) or Bayesian estimation of parameters are available, but they depend on an unknown normalizing constant, which may cause computational problems.

\section{PARAMETER ESTIMATION, MODEL I}

We will demonstrate the Takacz-Fiksel method of parameter estimation in the above model (Eq. 5). From the Georgii-Nguyen-Zessin formula (Eq. 2) we obtain the so called innovation

$$
\sum_{u \in X} q(u, X \backslash u)-\int_{Y} \lambda^{*}(X, u) q(u, X) d u
$$

which is a centered random variable. Using suitable test functions $q$ and setting innovations equal to zero leads to a system of equations for unknown parameters $\kappa, a, z$ given that $r$ and $v$ are known. These latter parameters are observable, the length $r$ directly, the dominant direction $v$ by the methods of mode estimation. The asymptotic properties of this estimator are studied in Coeurjolly et al. (2012). We use test functions $N_{\mathbf{x}}(u), \cos (2(\phi-v)), 1$, respectively, where $u=(y, \phi)$. Dividing the first two equations by the third one we obtain a system of two equations for unknown $a, \kappa$ :

$$
\begin{aligned}
\frac{1}{n(\mathbf{x})} \sum_{u \in \mathbf{x}} N_{\mathbf{x} \backslash u}(u) & =\frac{\sum_{i=1}^{J} b\left(a, \kappa, \mathbf{x}, u_{i}\right) N_{\mathbf{x}}\left(u_{i}\right)}{\sum_{i=1}^{J} b\left(a, \kappa, \mathbf{x}, u_{i}\right)}, \\
\frac{1}{n(\mathbf{x})} \sum_{u \in \mathbf{x}} \cos (2(\phi-v)) & =
\end{aligned}
$$

$$
=\frac{\sum_{i=1}^{J} b\left(a, \kappa, \mathbf{x}, u_{i}\right)\left(\cos \left(2\left(\phi_{i}-v\right)\right)\right)}{\sum_{i=1}^{J} b\left(a, \kappa, \mathbf{x}, u_{i}\right)},
$$

where

$$
b\left(a, \kappa, \mathbf{x}, u_{i}\right)=\exp \left(a N_{\mathbf{x}}\left(u_{i}\right)+\kappa \cos \left(2\left(\phi_{i}-v\right)\right)\right) .
$$

On the left hand side of the equations we have statistics of the data $\mathbf{x}$. Integrals on the right hand side are approximated by sums, using simulations of additional segments $u_{i}=\left(y_{i}, \phi_{i}\right), i=1, \ldots, J$, of fixed length $r$ from the uniform distribution on $Y$. Having solved (numerically) the above system of two equations we estimate the third parameter $z$ as

$$
z=\left(\frac{|B|}{n(\mathbf{x}) I_{0}(\kappa) J} \sum_{i=1}^{J} b\left(a, \kappa, \mathbf{x}, u_{i}\right)\right)^{-1} .
$$

\section{THE SEGMENT PROCESS WITH REFERENCE LENGTH DISTRIBUTION}

Next we extend the previous model by dealing also with a random segment length. The shape of the window and the location of segments take into account the intended biological application. Consider an ellipse $B \subset \mathbb{R}^{2}$ centred in the origin, with axes lengths $e_{1} \geq$ $e_{2}>0$ and area $|B|=\pi e_{1} e_{2} / 4$. Let $L_{o}=\left(0, e_{1}\right]$ be the interval of possible segment lengths, then

$$
Y=B \times L_{o} \times[0, \pi)
$$

is the space of segments $u=(s, r, \phi)$ which have centre $s$, length $r=l(u)$ and axial direction $\phi$.

Let the Poisson segment process $\eta(s)$ have the intensity measure $\lambda$ on $Y$ of the form

$$
\lambda(\mathrm{d}(y, r, \phi))=\frac{s}{e_{1} \pi} \mathrm{d} y \mathrm{~d} r \mathrm{~d} \phi
$$

for a constant $s>0$. Let the segment process $X$ have a density $p$ with respect to $\eta(1)$, model II:

$$
p(\mathbf{x})=c 1_{[\mathbf{x} \subset B]} \exp (b D(\mathbf{x})) z^{n(\mathbf{x})} \prod_{x_{i} \in \mathbf{x}} g\left(\frac{r_{i}}{e_{1}}\right),
$$

with parameters $b>0, z>0, n(\mathbf{x})$ is the total number of segments in $\mathbf{x}, c$ is the normalizing constant, $r_{i}$ is the length of $i$-th segment $x_{i}, g$ is a reference probability density on $L_{o}$ and

$$
D(\mathbf{x})=\sum_{u \in \mathbf{x}} d(u), \quad d(u)=\max _{w \in u} \frac{\|w\|}{e_{1}} .
$$

For $u \subset B$ we have $d(u) \in\left(0, \frac{1}{2}\right)$ which reflects the distance of the most distant point of the segment $u$ from 
the centre of the cell. The corresponding conditional intensity is

$$
\lambda^{*}(\mathbf{x}, u)=1_{[\mathbf{x} \cup u \subset B]} z g\left(\frac{r}{e_{1}}\right) \exp (b d(u)) .
$$

Example: We use the reference length density $g$ of the beta distribution with parameters $\alpha, \beta>0$

$$
g(r)=\frac{r^{\alpha-1}(1-r)^{\beta-1}}{B(\alpha, \beta)}, \quad r \in(0,1),
$$

with the beta function in the denominator. It is a natural unimodal distribution where two parameters enable the shift of the mode along the interval and also flexibility of the variance.

\section{PARAMETER ESTIMATION, MODEL II}

For the parameter estimation we suggest again the Takacz-Fiksel method. The parameters $e_{1}, e_{2}$ are known as described in the subsection on cell shape. Using four test functions

$$
d(u), \quad \log \left(\frac{l(u)}{e_{1}}\right), \quad \log \left(1-\frac{l(u)}{e_{1}}\right), \quad 1,
$$

and dividing the equations corresponding to the first three test functions by the equation corresponding to the fourth test function we obtain the system of three equations for unknown $b, \alpha, \beta$ :

$$
\begin{array}{r}
\frac{D(\mathbf{x})}{n(\mathbf{x})}=\frac{\sum_{i=1}^{J} \exp \left(b d\left(u_{i}\right)\right) g\left(\frac{r_{i}}{e_{1}}\right) d\left(u_{i}\right)}{\sum_{i=1}^{J} \exp \left(b d\left(u_{i}\right)\right) g\left(\frac{r_{i}}{e_{1}}\right)}, \\
\frac{\sum_{u \in \mathbf{x}} \log (l(u))}{n(\mathbf{x})}=\frac{\sum_{i=1}^{J} \exp \left(b d\left(u_{i}\right)\right) g\left(\frac{r_{i}}{e_{1}}\right) \log \left(r_{i}\right)}{\sum_{i=1}^{J} \exp \left(b d\left(u_{i}\right)\right) g\left(\frac{r_{i}}{e_{1}}\right)}, \\
\frac{\sum_{u \in \mathbf{x}} \log \left(e_{1}-l(u)\right)}{n(\mathbf{x})}= \\
=\frac{\sum_{i=1}^{J} \exp \left(b d\left(u_{i}\right)\right) g\left(\frac{r_{i}}{e_{1}}\right) \log \left(e_{1}-r_{i}\right)}{\sum_{i=1}^{J} \exp \left(b d\left(u_{i}\right)\right) g\left(\frac{r_{i}}{e_{1}}\right)},
\end{array}
$$

and finally we put the estimators of $b, \alpha, \beta$ in the equation for $z$ :

$$
z=\frac{M n(\mathbf{x})}{|B| \sum_{i=1}^{J} \exp \left(b d\left(u_{i}\right)\right) g\left(\frac{r_{i}}{e_{1}}\right)} .
$$

The segments $u_{i}=\left(y_{i}, r_{i}, \phi_{i}\right)$ are generated from the uniform distribution on $Y, i=1, \ldots, M$ and only $J$ of them which lie completely in $B$ are used.

\section{TESTING OF THE FIT OF THE MODEL}

Once we have estimated parameters of the model from the data, it is necessary to test whether the model fits the data well. Monte Carlo tests are common in spatial statistics, which are based on some scalar or functional test statistics of the data pattern (Møller and Waagepetersen, 2004). Then we simulate realizations of the model based on estimated parameters, and find upper and lower limits of values of estimated test statistics. In the case of test functions the envelopes formed by pointwise minima and maxima are plotted and it is evaluated how well the test function estimated from the data falls between the envelopes. Various designs of these methods are developed in Myllymäki et al. (2015).

In the present paper we restrict ourselves to scalar test statistics. Let $T_{i}, i=1, \ldots, n$, be a random sample of a statistic $T$,

$$
T_{l}=\min \left(T_{1}, \ldots, T_{n}\right), \quad T_{u}=\max \left(T_{1}, \ldots, T_{n}\right),
$$

obtained from independent simulations of the model with estimated parameters, and $\hat{T}$ is the observed value from the data. If $\hat{T}>T_{u}$ or $\hat{T}<T_{l}$ we reject the hypothesis that the data come from the model. The significance level is unknown since the testing procedure is not independent of the estimation procedure. Using the double Monte Carlo procedure from Dao and Genton (2014) it is possible to avoid this problem and reach the prescribed significance level which is computationally demanding.

Consider the null hypothesis that the segment directions $a_{1}, \ldots, a_{n}$ come from uniform directional distribution. Alternative hypothesis says that this is not the case. Axial data come from the interval $[0,180]$ in degrees. Let $a_{(1)} \leq a_{(2)} \leq \cdots \leq a_{(n)}$ be the ordered sample. Evaluate

$$
V_{n}=\max _{i=1, \ldots, n}\left(\frac{a_{(i)}}{180}-\frac{i}{n}\right)-\min _{i=1, \ldots, n}\left(\frac{a_{(i)}}{180}-\frac{i}{n}\right)+\frac{1}{n}
$$

and the modified Kuiper's test statistic (Mardia and Jupp, 1999) is

$$
V(\mathbf{x})=V_{n}\left(\sqrt{n}+0.155+\frac{0.24}{\sqrt{n}}\right) .
$$

Critical values for the test are tabulated, but data $\mathbf{x}$ from a single cell are not independent, therefore also here the Monte-Carlo approach described above has to be used. Having in mind that model II is isotropic on a circle, we consider the data $\mathbf{x}_{c}$ transformed from the elliptic cell shape onto a circle $B_{c}$ by means of new planar coordinates

$$
x^{\prime}=\frac{e_{2}}{e_{1}} x, \quad y^{\prime}=y .
$$

Then we pose a null hypothesis that $\mathbf{x}_{c}$ has uniform directional distribution and use the above described modified Kuiper's test. 


\section{RESULTS}

\section{SIMULATION RESULTS}

For a numerical demonstration of the TakaczFiksel method we simulated $n=100$ realizations of the segment process on $[0,1]^{2}$ of both model I (with parameters $v=\pi / 2, z=1000, a=-3, \kappa=$ $1, r=0.06$ ) and model II (with parameters $e_{1}=$ $e_{2}=1, z=100, b=0.5, \alpha=3, \beta=3$ ). Finding all intersections of a segment system is a problem from computational geometry. One can use the BentleyOttmann algorithm, (Bentley and Ottmann, 1979). In Fig.3 we demonstrate how model I depends on the interaction parameter $a$. The Takacz-Fiksel estimators of $(a, z, \kappa)$ (model I) and of $(z, b, \alpha, \beta)$ (model II) were evaluated with $J=1000$ for each realization. Empirical means and standard deviations (sd) of the estimators are printed in Table 1. It should be mentioned that in practice we have typically a single realization, while here we simulate one hundred independent realizations.
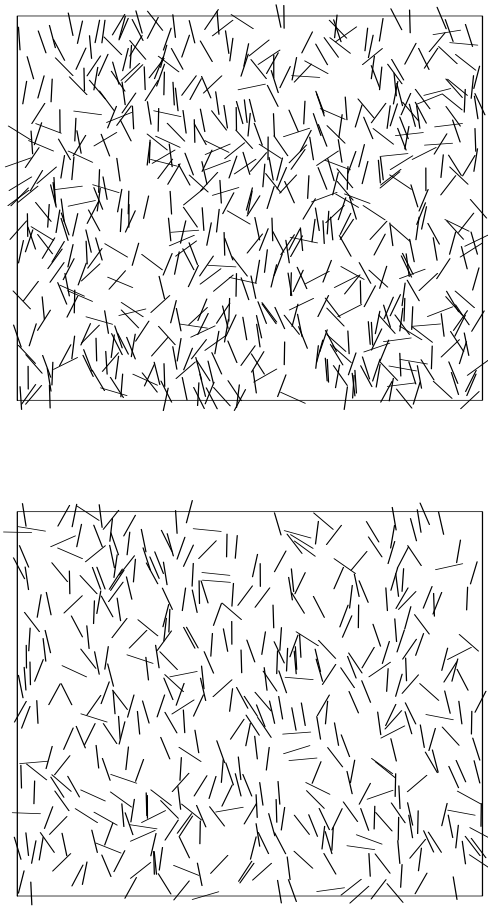

Fig. 3. Simulated realizations of model I segment processes on $[0,1]^{2}$ with parameters $v=\pi / 2, z=$ $1000, \kappa=1, r=0.06$, in the upper pattern we have $a=-0.6$ and statistics $n(\mathbf{x})=624, N(\mathbf{x})=204$. In the lower pattern we have $a=-3$ (more repulsion) and statistics $n(\mathbf{x})=433, N(\mathbf{x})=5$.
Table 1. Means and standard deviations (sd) of TakaczFiksel estimates from 100 simulations on $[0,1]^{2}$ of model I (with observable parameters $\mu=\pi / 2, r=$ 0.06 ) and model II (with $e_{1}=e_{2}=1$ ). The true values of estimated parameters are in the table.

\begin{tabular}{cccc}
\hline Model I & true & mean & sd \\
\hline$a$ & -3 & -2.998 & 0.299 \\
$\kappa$ & 1 & 0.999 & 0.078 \\
$z$ & 1000 & 1008.9 & 64.2 \\
\hline Model II & true & mean & sd \\
\hline$b$ & 0.5 & 0.525 & 0.463 \\
$\alpha$ & 3 & 3.089 & 0.296 \\
$\beta$ & 3 & 3.147 & 0.458 \\
$z$ & 100 & 104.32 & 40.25 \\
\hline
\end{tabular}

\section{REAL DATA RESULTS}

In Fig. 4 there are twenty cells from group $G 1$ for further analysis. Those cells were selected which fit the ellipsoidal shape best. The cell numbers and the relative amount of pixels outside the ellipse are given in Table 2.

Table 2. Shape description: the numbers of cells from group G1 investigated and their area outside ellipse $(A O E)$ in percent of pixels.

\begin{tabular}{cccc}
\hline No. & AOE [\%] & No. & AOE [\%] \\
\hline 001 & 3.03 & 043 & 5.87 \\
002 & 4.98 & 049 & 4.63 \\
005 & 7.96 & 055 & 3.61 \\
006 & 5.76 & 059 & 2.78 \\
018 & 6.91 & 060 & 5.00 \\
019 & 8.39 & 064 & 6.99 \\
020 & 7.20 & 092 & 6.98 \\
030 & 9.75 & 093 & 7.01 \\
031 & 7.75 & 127 & 5.59 \\
034 & 7.71 & 131 & 5.50 \\
\hline
\end{tabular}




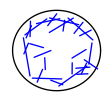

1

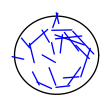

6

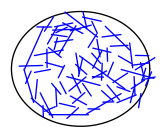

20

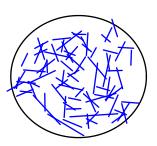

34

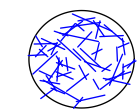

55
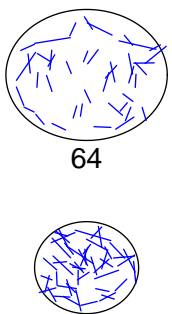

127

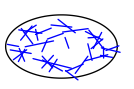

2

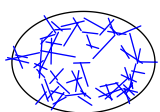

18

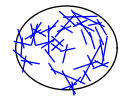

30

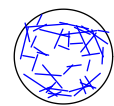

43

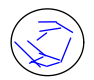

59

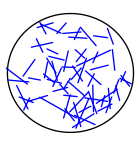

92

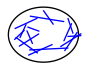

131

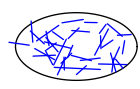

5

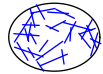

19

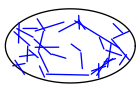

31

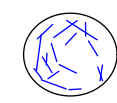

49

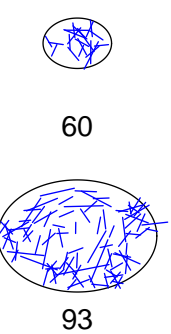

93
Finally we used the modified Kuiper's statistics (10) to the transformed data $\mathbf{x}_{c}$ in (11) and the result is in Fig. 6 . For one cell the test statistic lies outside the interval and in this case we reject the null hypothesis.
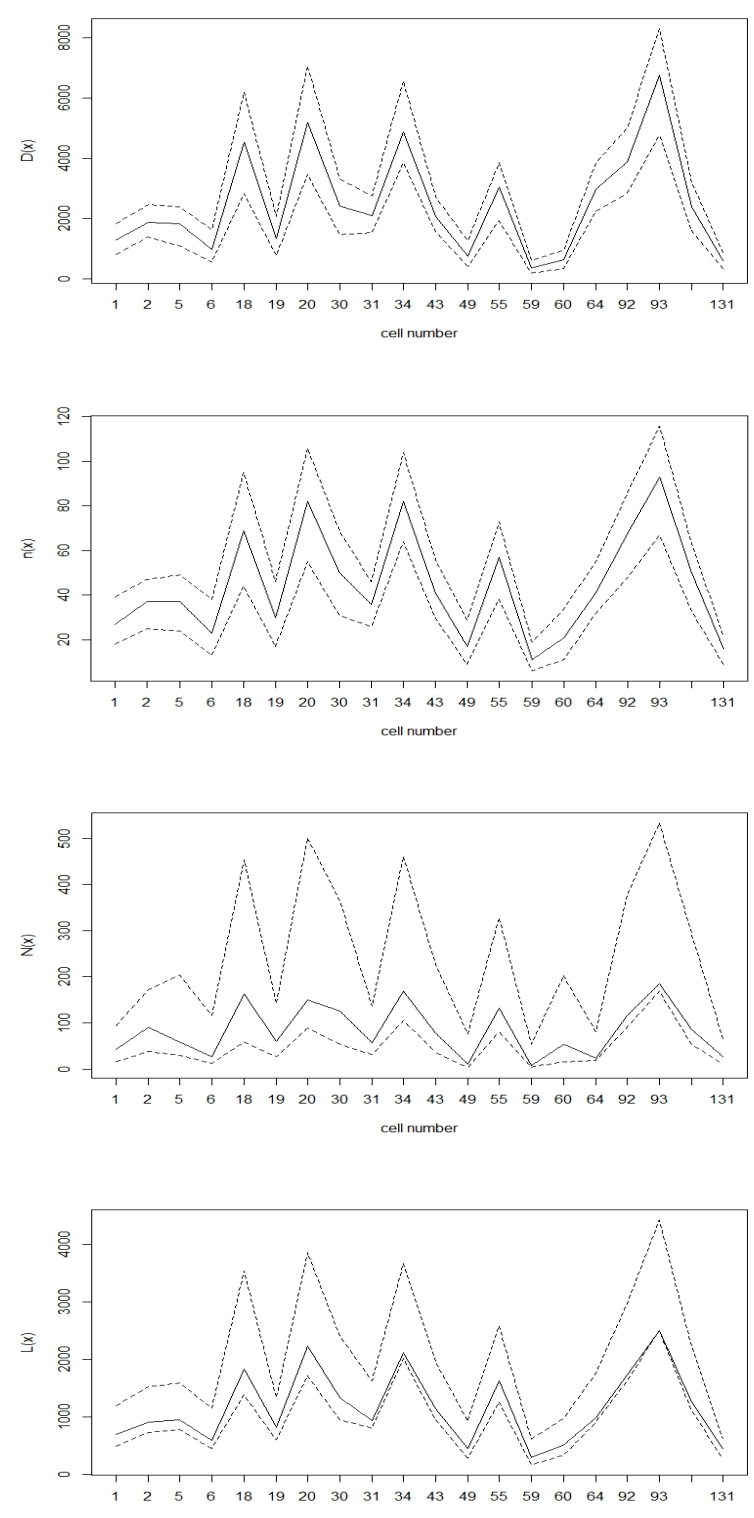

cell number

Fig. 5. The result of model II testing for the statistics $D(\mathbf{x}), n(\mathbf{x}), N(\mathbf{x}), L(\mathbf{x})$ for analysed segment systems corresponding to selected cells from group $G 1$. On the horizontal axis there are numbers of cells. The bounds (8) are plotted and joined by dashed lines, the values of the test statistics from real data lie between them (joined by a full line). This does not lead to the rejection of model II fit. 
Table 3. The results of the Takacz-Fiksel estimator with reference beta length distribution, model II. The columns involve subsequently: the cell number, the axes lengths $e_{1}, e_{2}$ (in pixels, where 1 pixel $=0.32 \mu \mathrm{m}$ ), estimated parameters $z, b, \alpha, \beta$ and the ratio $I=\frac{n(\mathbf{x})}{e_{1} e_{2}}$ which is proportional to number density of segments.

\begin{tabular}{rrrrrccc}
\hline No & $e_{1}$ & $e_{2}$ & $z .10^{3}$ & $b .10^{2}$ & $\alpha$ & $\beta$ & $I .10^{2}$ \\
\hline 001 & 59 & 54 & 0.46 & 4.99 & 1.76 & 4.27 & 0.83 \\
002 & 76 & 43 & 1.67 & 2.88 & 1.58 & 4.35 & 1.13 \\
005 & 83 & 46 & 4.49 & 0.45 & 1.59 & 4.50 & 0.97 \\
006 & 57 & 53 & 1.98 & 1.70 & 2.00 & 4.47 & 0.76 \\
018 & 97 & 69 & 1.77 & 1.65 & 1.34 & 4.95 & 1.03 \\
019 & 62 & 46 & 1.12 & 3.97 & 1.87 & 4.05 & 1.05 \\
020 & 94 & 78 & 4.07 & 0.41 & 1.41 & 4.67 & 1.12 \\
030 & 69 & 57 & 3.54 & 1.13 & 1.75 & 4.38 & 1.27 \\
031 & 88 & 50 & 1.18 & 2.40 & 1.44 & 4.46 & 0.81 \\
034 & 92 & 83 & 9.44 & -1.05 & 1.43 & 4.71 & 1.07 \\
043 & 67 & 64 & 2.43 & 1.38 & 1.81 & 4.52 & 0.95 \\
049 & 63 & 56 & 2.54 & -0.02 & 1.86 & 4.34 & 0.48 \\
055 & 72 & 66 & 2.25 & 1.85 & 1.91 & 5.22 & 1.20 \\
059 & 49 & 45 & 19.03 & -5.41 & 2.74 & 4.42 & 0.49 \\
060 & 46 & 34 & 19.10 & -2.57 & 2.84 & 4.92 & 1.31 \\
064 & 109 & 89 & 1.48 & 0.28 & 1.29 & 5.75 & 0.42 \\
092 & 86 & 80 & 7.89 & -1.00 & 1.69 & 5.91 & 0.98 \\
093 & 108 & 76 & 1.98 & 1.42 & 1.25 & 4.63 & 1.13 \\
127 & 70 & 65 & 7.63 & -0.60 & 1.65 & 4.28 & 1.13 \\
131 & 48 & 34 & 0.14 & 10.26 & 5.73 & 12.3 & 0.90 \\
\hline
\end{tabular}

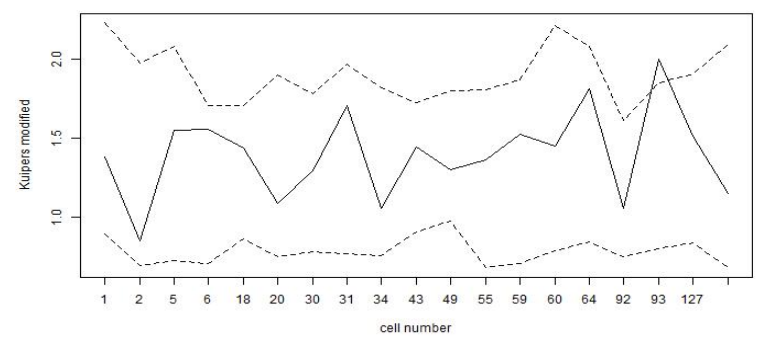

Fig. 6. The result of testing the uniformity of directional distribution for the data $\mathbf{x}_{c}$ transformed to a circle. The full line corresponds to $V\left(\mathbf{x}_{c}\right), c f .(10)$, the bounds (8) are based on 20 simulations of the model on a circle with parameters estimated from transformed data. Cell 19 is omitted since the estimator has not been achieved, for the cell 93 we observe that $V\left(\mathbf{x}_{c}\right)$ does not lie between the bounds which leads to the rejection of null hypothesis.

\section{DISCUSSION}

As written in the Introduction, the paper has two aims, a mathematical and an applied one. The segment process having a density with respect to the Poisson process and reference directional and/or length distributions presents a new model for segment systems on a bounded set which may posses interactions. More complex models can be built by using joint direction-length distribution models, but in fact model II is of this kind where the directional distribution is uniform. It should be mentioned that generally the reference distribution need not coincide with the observed distribution. The model $\mathrm{I}$ is a Gibbs type homogeneous process while model II is an inhomogeneous Poisson process. We suggested the parameter estimator based on Takacz-Fiksel method for both models, the estimating equations were solved numerically using the Nelder-Mead method. First we showed the capabilities of the estimation procedure in simulated segment systems.

Model I was introduced for simulation and demonstration purposes, because of its homogeneity it arised not to be useful for the modeling of real data from hMSCs. Theferore we tried to apply the model II to real data of stress fibres observed by fluorescence imaging and transformed into segment systems. In model II we involve a special statistics $D(\mathbf{x})$ which performs quite well. The negative values of the corresponding parameter $b$ for cells 34,49,59,60,92,127 (cf. Table 3) correspond to a uniform distribution of short filaments across the cell or even tendency to cluster around the centre. Positive values in other cases correspond to a typical accumulation more close to the boundary than to the centre of the cell. The beta distribution of the length is also stable in parameters $\alpha, \beta$ (with the exception of cell 131). Positive results of the degreeof-fit test in Fig.5 do not yet mean that the data completely correspond to the model since the test is conservative. Moreover functional characteristics (like the contact distribution function) could be implemented as descriptors of spatial distribution. Nevertheless all the presented arguments together make the model II interesting and valuable for the underlying biological problem.

\section{ACKNOWLEDGEMENT}

The research was supported by the Czech Science Foundation, project no. 16-03708S. 


\section{REFERENCES}

Baddeley A (2007). Spatial point processes and their applications. In: Weil W ed. Stochastic geometry. Lect Not Math 1892:1-75.

Bentley JL, Ottmann TA (1979). Algorithms for reporting and counting geometric intersections. IEEE Transac Comput C28(9):643-47.

Chiu B, Stoyan D, Kendall WS, Mecke J (2013). Stochastic Geometry and Its Applications. 3rd Ed. New York: Wiley.

Coeurjolly JF, Dereudre D, Drouilhet R, Lavancier F (2012). Takacs-Fiksel Method for Stationary Marked Gibbs Point Processes. Scand J Statist 39:416-43.

Dao A, Genton, MG (2014). A Monte Carlo adjusted goodness-of-fit test for parametric models describing spatial point patterns. J Comput Graph Statist 23:497517.

Dereudre D, Lavancier F, Helisova K (2014). Estimation of the intensity parameter of the germ-grain Quermassinteraction model when the number of germs is not observed. Scand J Statist 41:809-29.

Eltzner B, Wollnik C, Gottschlich C, Huckemann S, and Rehfeldt F (2016). The filament sensor for near realtime detection of cytoskeletal fiber structures. PLOS ONE 10(5):e0126346.

Engler AJ, Sen S, Sweeney HL, Discher DE (2006). Matrix elasticity directs stem cell lineage specification. Cell 126(4):677-89.
Geyer CJ, Møller J (1994). Simulation procedures and likelihood inference for spatial point processes. Scand J Statist 21:359-73.

Mardia KV, Jupp PE (1999). Directional Statistics. New York: Wiley.

Møller J, Helisova K (2008). Power diagrams and interaction process for unions of discs. Adv Appl Probab 40:321-47.

Møller J, Helisova K (2010). Likelihood inference for unions of interacting discs. Scand J Statist 37:365-81.

Møller J, Waagepetersen R (2004). Statistical Inference and Simulation for Spatial Point Processes. Singapore: CRC Press.

Møller J, Waagepetersen R (2007). Modern Statistics for Spatial Point Processes. Scand J Statist 34:643-84.

Myllymäki M, Grabarnik P, Seijo H, Stoyan D (2015). Deviation test construction and power comparison for marked spatial point patterns. Spat Statist 11:19-34.

Pawlas, Z (2014). Self-crossing points of a line segment process. Method Comput Appl Probab 16:295-309.

Večeřa J, Beneš V (2016). Interaction processes for unions of facets, the asymptotic behaviour with increasing intensity. Method Comput Appl Probab 18(4):1217-39.

Večeřa J (2016). Central limit theorem for Gibbsian Ustatistics of facet processes. Appl Math 61(4):423-41.

Zemel A, Rehfeldt F, Brown AEX, Discher DE, Safran SA (2010). Optimal matrix rigidity for stress-fibre polarization in stem cells. Nature Physics 6:468-73. 